\title{
Falls in hospital and new placement in a nursing home among older people hospitalized with acute illness
}

\author{
David Basic' \\ Tabitha J Hartwell ${ }^{2}$ \\ 'Department of Geriatric Medicine, \\ Liverpool Hospital, Sydney, NSW, \\ Australia; ${ }^{2}$ Department of Geriatric \\ Medicine and Rehabilitation, \\ Shoalhaven District Memorial \\ Hospital, Nowra, NSW, Australia
}

This article was published in the following Dove Press journal:

Clinical Interventions in Aging

14 October 2015

Number of times this article has been viewed

Purpose: To examine the association between falls in hospital and new placement in a nursing home among older people hospitalized with acute illness.

Materials and methods: This prospective cohort study of 2,945 consecutive patients discharged alive from an acute geriatric medicine service used multivariate logistic regression to model the association between one or more falls and nursing home placement (primary analysis). Secondary analyses stratified falls by injury and occurrence of multiple falls. Demographic, medical, and frailty measures were considered in adjusted models.

Results: The mean age of all patients was $82.8 \pm 7.6$ years and $94 \%$ were admitted through the emergency department. During a median length of stay (LOS) of 11 days, 257 (8.7\%) patients had a fall. Of these, $66(25.7 \%)$ sustained an injury and $53(20.6 \%)$ had two or more falls. Compared with nonfallers, fallers were more likely to be placed in a nursing home (odds ratio [OR]: 2.03, 95\% confidence interval [CI]: 1.37-3.00), after adjustment for age, sex, frailty, and selected medical variables (including dementia and delirium). Patients without injury (OR: 1.83, 95\% CI: 1.17-2.85) and those with injury (OR: 2.35, 95\% CI: 1.15-4.77) were also more likely to be placed. Patients who fell had a longer LOS (median 19 days vs 10 days; $P<0.001$ ).

Conclusion: This study of older people in acute care shows that falls in the hospital are significantly associated with new placement in a nursing home. Given the predominantly negative experiences and the financial costs associated with placement in a nursing home, fall prevention should be a high priority in older people hospitalized with acute illness.

Keywords: aged, inpatients, falls, nursing homes

\section{Introduction}

As the population ages, the numbers in the oldest age groups will increase the fastest. ${ }^{1}$ The prevalence of chronic diseases will increase exponentially, and most hospital beds in the developed nations will be occupied by older people, many of whom will be admitted because of delirium, functional impairment, deconditioning, gait abnormality, and falls.

Most falls in hospital result from synergistic interactions between person-specific risk factors, the physical environment, the riskiness of a person's behavior, and the interactions between the patient and hospital staff. ${ }^{2}$ During hospitalization for acute illness, these synergistic interactions are magnified and falls become a major concern for older people, their carers, the hospital, and the health care system.

Falls in the hospital are common, with rates ranging from $1.1^{3}$ to $15.7^{4}$ falls per 1,000 occupied bed days. While higher rates are reported from aged care, rehabilitation, neurology, and aged care psychiatry wards, ${ }^{2}$ few clinical areas are spared. Between 
$30 \%$ and $50 \%$ of falls during hospitalization result in injury. Fractures occur in 1\%-3\% of patients and hip fractures in $1.1 \%-2 \% .^{2}$ Even minor soft tissue injuries can cause significant impairment in older people with poor functional reserve. ${ }^{2}$ Recent systematic reviews suggest that $20 \%-30 \%$ of hospital falls can be prevented. ${ }^{5,6}$ Although this reduction may seem modest, the cost savings are potentially large. Nursing home care is also expensive and hence further cost savings will ensue if it can be prevented.

Although positive experiences in nursing homes can occur, negative experiences predominate in many countries. People in nursing homes frequently voice concerns about lack of privacy, autonomy, and dignity, ${ }^{7}$ which together with difficulty in forming positive relationships with others, ${ }^{8}$ promote feelings of helplessness and loneliness. ${ }^{9}$ Although nursing home care is inevitable in some, most older people prefer to remain in their own home. In Australia, older people discharged from hospital rarely transition through a nursing home before subsequent return home. Most who are discharged to a nursing home stay there permanently.

Few studies have evaluated the association between falls in hospital and nursing home placement. Murray et $\mathrm{al}^{10}$ reported that $33 \%$ of patients with proximal femoral fractures sustained in the hospital were newly discharged to a nursing home. Although the discharge destinations were not stated in detail, Foss et $\mathrm{al}^{11}$ found that $66 \%$ of patients with hospital-acquired hip fractures were not discharged to their original residence. In one study of patients discharged from a nonacute geriatric hospital, falls were independently associated with the risk of nursing home placement. ${ }^{12}$ In another nonacute study, recurrent fallers were significantly more likely to be discharged to a nursing home (compared to nonfallers and single fallers). ${ }^{13}$ There are no publications on this topic in older people discharged from acute care, particularly in more contemporary settings.

The primary aim of this study was to evaluate the association between falls in hospital and new placement in a nursing home among older people hospitalized with acute illness. The secondary aim was to study the effect of fall-related injury, or its absence, on this association. A better understanding of falls and their potential consequences may inform both clinicians and health service planners.

\section{Materials and methods}

\section{Study setting and participants}

This prospective study was undertaken at Liverpool Hospital, a busy university hospital in South-Western Sydney, Australia. Potential participants were 4,182 consecutive patients admitted to the acute geriatric medicine service between January 1, 2010 and December 31, 2012. Patients admitted from a nursing home, and those who died in the hospital were excluded, leaving 2,945 actual participants. Most were admitted based on targeting criteria that included delirium, deconditioning, functional impairment, gait abnormality and falls, multiple medical diagnoses, polypharmacy, malnutrition, and multiple unplanned admissions. ${ }^{14}$ The South Western Sydney Local Health District Human Research Ethics Committee provided ethics approval for the study. Patient consent was not required as the study was deemed to be of low and negligible risk.

\section{Measures}

A multidisciplinary team, comprising medical, nursing, and allied health professionals, comprehensively assessed all patients. We collected data on patient demographics (including admission and discharge domicile), referral source, medical diagnoses (including those acquired in hospital), premorbid frailty, in-hospital mortality, length of stay (LOS), and in-hospital falls.

The inpatient multidisciplinary team recommended nursing home placement for those who needed higher levels of care. Approval for placement was independently undertaken by the Aged Care Assessment Team, an external team delegated by the Australian Minister of Health to approve nursing home placement when needed. Both the multidisciplinary team and the Aged Care Assessment Team made their recommendations in discussion with the patient and the family (or nonfamily carers) whenever possible.

Based on version 5.1 of the Australian Refined Diagnosis Related Groups classification system, medical diagnoses were grouped into 21 categories (eg, neurological), with each category containing up to 17 individual diagnoses, for a total of 177 diagnoses. They were chosen in consensus with other geriatricians at the hospital and reflected diagnoses relevant to geriatric medicine. We coded up to 15 active diagnoses per patient (those impacting on physical, social, or psychological function, or needing medication changes, investigations or increased monitoring to treat symptoms and guide management). While some diagnoses (eg, dysphagia) are more appropriately termed problems or syndromes, for consistency we refer to all as diagnoses. Premorbid frailty was defined as the level of frailty present 1 month before the admission to the hospital and was measured using the Canadian Study of Health and Aging Clinical Frailty Scale (CSHA-CFS). ${ }^{15}$ The CSHA-CFS is a subjective scale that relies on clinical judgment. It mixes items such as comorbidity, cognitive impairment, and disability to classify people into one of seven ordinal categories (Table 1). At the two 
Table I Canadian Study of Health and Aging Clinical Frailty Scale

\begin{tabular}{ll}
\hline Category & Category description \\
\hline $\mathrm{I}$ & Very fit (energetic, motivated, exercise regularly) \\
2 & Well (no active disease, less fit than category I) \\
3 & Well, with treated comorbid disease \\
4 & Apparently vulnerable (not frankly dependent, disease \\
& symptoms, slowed up) \\
5 & Mildly frail (limited dependence in IADL) \\
7 & Moderately frail (help needed with ADL and IADL) \\
& Severely frail (completely dependent in ADL, or \\
\hline
\end{tabular}

Abbreviations: IADL, instrumental activities of daily living; ADL, basic activities of daily living.

extremes of the scale, those in category one are very fit, energetic, and motivated, whereas those in category seven are completely dependent in the activities of daily living or are terminally ill.

Except for falls, the attending geriatricians collated and prospectively entered all data into a clinical database housed on a network drive. Falls data were retrospectively and manually retrieved from a mandatory institutional incident reporting database (by author DB). A fall was defined as an unplanned descent to the floor, or extension of the floor, irrespective of the cause. Assisted falls, where a staff member reduced the impact of a fall, were included.

\section{Statistical analysis}

Multiple logistic regression was used to model the probability of nursing home placement. The study variable, fall in the hospital, and risk factors in the literature (dementia, delirium, age, and sex $)^{12,16,17}$ were forced into all models. Other variables from our dataset were considered for inclusion based on biological plausibility, significance, and confounding (between falls and nursing home placement). These included behavioral and psychological symptoms of dementia (BPSD), deconditioning, severe malnutrition, presence of a pressure area (grade 2 or higher), cardiac failure, renal failure, intracranial hemorrhage, severe proximal myopathy, severe peripheral neuropathy, severe arthritis of the hip and knee, fracture of the pelvis, vertebra and humerus, major depression, severe hearing and visual impairment, urine retention, Parkinson's disease, and frailty (CSHA-CFS). The primary analysis considered all falls (one or more falls). Secondary analyses stratified falls according to fall-related injury and the occurrence of more than one fall (multiple falls). The degree of confounding exerted by a variable was measured by calculating the change in the value of the parameter estimate for the study factor (fall in the hospital) when the variable was excluded from the analysis. A $10 \%$ or greater change in the parameter estimate was needed for a confounder to be retained in the model. To evaluate whether the effect of falls on nursing home placement was modified by age, sex, dementia, delirium, and frailty (effect modification), we dichotomized these variables and created interaction terms for testing in the multivariate models. Age was dichotomized around its mean value (82.8 years), while frailty was dichotomized according to the presence of moderate-to-severe frailty (versus lesser frailty) (Table 1). Between-groups comparisons were tested using $t$-tests for continuous normally distributed variables, Wilcoxon ranksum tests for ordinal variables, and Fisher's exact tests for dichotomous variables. The linear correlation between variables was measured with Spearman's rank-order correlation coefficient. Analysis was performed using SAS, version 9.3 (SAS Institute, Inc., Cary, NC, USA).

\section{Results}

\section{Characteristics of study participants}

The mean age of all participants was $82.8 \pm 7.6$ years, and $38.3 \%$ of the participants were male. Most (85.5\%) were admitted from community-based homes, including boarding homes, caravans, and independent living units. The study population was multicultural, with 1,542 (52.4\%) participants born in countries where English was not the predominant language spoken. Most (93.7\%) were admitted through the emergency department and were acutely unwell, with a median of seven active diagnoses. Table 2 shows the characteristics of the participants according to fall status.

\section{Falls, fall-related injuries, and length of stay}

During a median LOS of 11 days, 257 (8.7\%) patients had a fall. Of these, 204 (79.4\%) had a single fall, 33 (12.8\%) had two falls, $13(5.1 \%)$ had three falls, and 7 (2.7\%) had four or more falls. Sixty-six (25.7\%) patients sustained a fall-related injury. All five $(1.9 \%)$ of the severe injuries were fractures (two hip fractures and single fractures of the ankle, wrist, and vertebra). Although 15 patients sustained a head injury, all were minor in severity. Three patients had minor single joint injuries (involving the hip, shoulder, and elbow) and two had unspecified injuries to the sacrum and leg, none of which caused sustained functional impairment. All other injuries involved the skin. Most were small lacerations and only two needed multiple sutures.

Compared with nonfallers, patients who fell had significantly longer LOS (median 19 days vs 10 days; $P<0.001$ ). Patients who sustained an injury and those who fell more than once had median lengths of stay of 23 and 27 days, 
Table 2 Characteristics of study participants according to fall

\begin{tabular}{|c|c|c|c|c|c|}
\hline \multirow[t]{2}{*}{ Characteristic } & \multicolumn{2}{|l|}{ Fall } & \multirow[t]{2}{*}{ Characteristic } & \multicolumn{2}{|l|}{ Fall } \\
\hline & Yes, $\mathbf{N}=\mathbf{2 5 7}$ & No, $\mathbf{N}=2,688$ & & Yes, $\mathbf{N}=\mathbf{2 5 7}$ & No, $\mathbf{N}=\mathbf{2 , 6 8 8}$ \\
\hline Age (mean $\pm S D)$ & $82.3 \pm 7.4$ & $82.8 \pm 7.7$ & Intracranial hemorrhage (\%) & 2.7 & 0.7 \\
\hline Male sex (\%) & 54.5 & 36.8 & Knee arthritis (\%) & 4.3 & 2.7 \\
\hline Non-English-speaking COB (\%) & 52.9 & 52.3 & Hip arthritis (\%) & 3.9 & 2.9 \\
\hline English-speaking (\%) & 69.7 & 70.1 & Malnutrition (\%) & 9.7 & 7.0 \\
\hline Admission domicile (\%) & & & Dysphagia (\%) & 11.3 & 7.9 \\
\hline Community-based home & 82.1 & 85.9 & Cardiac failure (\%) & 16.0 & 16.6 \\
\hline Hostel & 17.9 & 14.1 & Myocardial ischemia (\%) & 12.1 & 15.7 \\
\hline Referral source (\%) & & & Renal failure (\%) & 25.7 & 26.1 \\
\hline Emergency department & 91.8 & 93.9 & COPD (\%) & 8.2 & 13.6 \\
\hline Consultation and transfer care & 6.2 & 4.7 & Proximal myopathy (\%) & 2.0 & 0.9 \\
\hline Other & 2.0 & 1.4 & Peripheral neuropathy (\%) & 3.9 & 3.4 \\
\hline CSHA-CFS category (\%) & & & Parkinson's disease (\%) & 3.5 & 3.2 \\
\hline I & 0.5 & 0.7 & Stroke (\%) & 7.8 & 5.2 \\
\hline 2 & 0.0 & 1.6 & Pelvic fracture (\%) & 2.0 & 3.3 \\
\hline 3 & 2.7 & 4.3 & Vertebral fracture (\%) & 5.1 & 4.4 \\
\hline 4 & 5.5 & 9.2 & Humeral fracture (\%) & 0.8 & 1.2 \\
\hline 5 & 22.8 & 27.9 & Fracture (\%) & 17.1 & 15.8 \\
\hline 6 & 57.1 & 43.3 & Infection (\%) & 61.5 & 56.3 \\
\hline 7 & 11.4 & 13.0 & Malignant neoplasm (\%) & 14.4 & 11.6 \\
\hline Dementia (\%) & 59.5 & 38.1 & Major depression (\%) & 3.9 & 2.8 \\
\hline Delirium (\%) & 51.0 & 29.4 & Hearing impairment (\%) & 3.9 & 3.2 \\
\hline BPSD (\%) & 11.7 & 5.6 & Visual impairment (\%) & 4.7 & 6.1 \\
\hline Deconditioning (\%) & 29.2 & 20.5 & Pressure area (\%) & 2.0 & 2.4 \\
\hline Urine retention (\%) & 17.5 & 9.7 & & & \\
\hline
\end{tabular}

Notes: CSHA-CFS data are missing in 38 (14.8\%) fallers and 288 (10.7\%) nonfallers. All other data are fully complete. Other source of referral to geriatrician refers to other hospitals and outpatient departments. The diagnoses displayed are those commonly seen in older inpatients (shown to further define the study population). Knee arthritis, hip arthritis, malnutrition, proximal myopathy, peripheral neuropathy, hearing impairment, and visual impairment refer to severe disease. Pressure area refers to grade 2 or higher. Abbreviations: SD, standard deviation; COB, country of birth; CSHA-CFS, Canadian Study of Health and Aging Clinical Frailty Scale; BPSD, behavioral and psychological symptoms of dementia; COPD, chronic obstructive pulmonary disease.

respectively (both $P<0.001$ ). Fewer patients who fell died in the hospital $(8.3 \%$ vs $11.9 \%, P=0.06, n=4,182)$. Two patients admitted from a nursing home died as a direct consequence of a fall, both from an intracranial hemorrhage.

\section{Falls and new nursing home placement}

A total of $311(10.6 \%)$ patients were newly placed in a nursing home. Patients who fell were more likely to be placed in a nursing home when compared to nonfallers (odds ratio [OR]: 2.81, 95\% confidence interval [CI]: 2.04-3.86), irrespective of the absence (OR: 2.63 , 95\% CI: 1.82-3.81) or presence (OR: 3.34, 95\% CI: 1.90-5.89) of injury or multiple falls (OR: 3.13, 95\% CI: 1.65-5.93) (all $P<0.001$ ).
Additional unadjusted data are shown in Table 3. Patients who fell and were placed had a median LOS of 21.5 days, several days longer than those who fell but returned home (median 19 days; $P=0.08$ ).

Table 4 shows the final logistic regression models for nursing home placement. Compared with nonfallers, fallers were significantly more likely to be placed in a nursing home. In the secondary multivariate analyses shown in Table 4, patients without injury and those with injury were also more likely to be placed. Multiple falls did not predict placement, most likely a consequence of statistical uncertainty due to few patients in this category needing placement (13 of 53).

Table 3 New placement in a nursing home according to fall, fall-related injury, and multiple falls

\begin{tabular}{|c|c|c|c|c|c|c|c|c|}
\hline \multirow[t]{3}{*}{ Fall } & \multicolumn{2}{|c|}{ One or more falls } & \multicolumn{2}{|c|}{ Falls without injury } & \multicolumn{2}{|c|}{ Falls with injury } & \multicolumn{2}{|c|}{ Multiple falls } \\
\hline & \multicolumn{2}{|l|}{ Placed } & \multicolumn{2}{|l|}{ Placed } & \multicolumn{2}{|l|}{ Placed } & \multicolumn{2}{|l|}{ Placed } \\
\hline & Yes & No & Yes & No & Yes & No & Yes & No \\
\hline Yes & $58(22.6)$ & $199(77.4)$ & $4 \mid(2 I .5)$ & $150(78.5)$ & $17(25.8)$ & $49(74.2)$ & $13(24.5)$ & $40(75.5)$ \\
\hline No & $253(9.4)$ & $2,435(90.6)$ & $253(9.4)$ & $2,435(90.6)$ & $253(9.4)$ & $2,435(90.6)$ & $253(9.4)$ & $2,435(90.6)$ \\
\hline
\end{tabular}

Note: Cross-tabulation data show the frequency and the row percent for each subset of falls. 
Table 4 Predictors of new placement in a nursing home according to fall, fall-related injury, and multiple falls

\begin{tabular}{|c|c|c|c|c|}
\hline \multirow[t]{2}{*}{ Predictor } & \multirow{2}{*}{$\frac{\text { Primary analysis }}{\text { One or more falls }}$} & \multicolumn{3}{|l|}{ Secondary analyses } \\
\hline & & Falls without injury & Falls with injury & Multiple falls \\
\hline$\overline{\text { Fall }}$ & $2.03(1.37-3.00)$ & $1.83(1.17-2.85)$ & $2.35(1.15-4.77)$ & I.5I (0.69-3.30) \\
\hline Dementia & $1.97(1.46-2.67)$ & $1.93(1.42-2.62)$ & $1.97(1.43-2.72)$ & $1.95(1.41-2.69)$ \\
\hline Delirium & $0.73(0.55-0.98)$ & $0.76(0.56-1.02)$ & $0.73(0.53-1.00)$ & $0.76(0.56-1.04)$ \\
\hline Age & $1.02(1.00-1.04)$ & $1.02(1.00-1.04)$ & $1.02(1.00-1.04)$ & $1.02(1.00-1.04)$ \\
\hline Male sex & $1.67(1.27-2.21)$ & $1.66(1.25-2.20)$ & $1.75(1.31-2.35)$ & $1.70(1.27-2.29)$ \\
\hline CSHA-CFS & $3.39(2.74-4.18)$ & $3.37(2.72-4.18)$ & $3.20(2.57-3.98)$ & $3.24(2.60-4.05)$ \\
\hline BPSD & $2.53(1.69-3.81)$ & $2.61(1.73-3.96)$ & $2.53(1.63-3.93)$ & $2.62(1.69-4.07)$ \\
\hline Urine retention & $2.10(1.47-3.01)$ & $2.13(1.48-3.07)$ & $2.08(1.4 I-3.06)$ & $1.97(1.34-2.90)$ \\
\hline Knee arthritis (severe) & $2.36(1.20-4.62)$ & $2.42(1.21-4.86)$ & $2.39(1.20-4.79)$ & $2.37(1.19-4.74)$ \\
\hline Deconditioning & $1.43(1.04-1.96)$ & $1.52(1.10-2.10)$ & $1.54(1.10-2.15)$ & $1.73(1.24-2.4 \mid)$ \\
\hline Intracranial hemorrhage & & $2.93(1.08-7.90)$ & $3.68(1.33-10.14)$ & $3.89(1.4 I-10.75)$ \\
\hline
\end{tabular}

Notes: Dementia, delirium, age, and sex were forced into all models. Age was not significant in falls with injury $(P=0.09)$ and multiple falls $(P=0.06)$ models. Spearman correlation coefficients between dementia and BPSD $=0.26$, between delirium and dementia $=0.25$, and between delirium and CSHA-CFS $=0.24$. Data shown as OR (95\% Cl).

Abbreviations: OR, odds ratio; Cl, confidence interval; CSHA-CFS, Canadian Study of Health and Aging Clinical Frailty Scale; BPSD, behavioral and psychological symptoms of dementia.

The effect of falls on nursing home placement was not modified by age, sex, dementia, delirium, or frailty in any of the subsets of falls shown in Table 4.

\section{Discussion}

This large cohort study of older people hospitalized with acute illness shows that patients who fell in the hospital were significantly more likely to be newly placed in a nursing home, irrespective of the absence or presence of injury. This relationship remained strong even after adjustment for established risk factors for placement and appropriately selected variables, including dementia, BPSD, and frailty.

Several mechanisms may drive the relationship between falls and nursing home placement. In a community-dwelling population, Tinetti and Williams ${ }^{18}$ raised the possibility that falling is merely a marker for frailty in people already at risk of institutional care. In a similar population, Dunn et al ${ }^{19}$ found a weaker relationship between falls and placement after adjustment for the ability to perform the activities of daily living. While explanatory variables may differ in effect and magnitude in community-dwelling populations, our data show a strong association between falls and placement even after adjustment for frailty, measured using the CSHA-CFS. Among its domains, the CSHA-CFS includes both instrumental and basic activities of daily living. ${ }^{15}$

Injury is also an important mechanism for placement. ${ }^{10,11,18}$ Fall-related injuries, including supposedly minor soft tissue injuries, can cause considerable functional impairment in older people with poor functional reserve. ${ }^{2}$ These injuries may instigate a negative cycle whereby fear of falling causes limitation of activity and further losses of strength and independence, ${ }^{2}$ beginning, in some, a process that ultimately culminates in nursing home placement. While injury is an important mechanism, our data show that patients without injury were similarly likely to be placed, raising the possibility that fear of falling may be an important factor driving placement in many patients.

Many older persons now access a range of services that do not involve inpatient care, including those provided by hospital in the home programs, ambulatory care departments, outpatient clinics, and day hospitals. Systems of inpatient care also continue to evolve with the emergence of short-stay units as alternatives to standard hospitalization. ${ }^{20-22}$ Older people needing standard hospital care have an increasing burden of disease and more complex needs. These issues cast some doubt on the relevance of older studies of falls and placement, particularly those conducted in nonacute hospitals, ${ }^{12,13}$ to more contemporary settings. Modern-day studies are better placed to inform policy makers and service providers on the potential impact of falls, an ongoing concern given the increasing age and complexity of older inpatients juxtaposed against budget cuts. To the best of our knowledge, our study is the first of its kind in older people discharged from acute care.

Table 4 suggests that delirium protects against nursing home placement. When dementia and frailty are removed from the models in sensitivity analyses, delirium predicts nursing home placement in each subset of falls (data not shown, all $P<0.05)$. Delirium also predicts placement in univariate analysis $(P<0.001)$. This suggests that concomitant dementia and frailty may determine the need for placement in many patients with delirium. Patients in acute care who 
are neither frail nor demented and who survive their acute illness should be able to return home in most cases. The correlations between delirium and dementia, and between delirium and frailty (CSHA-CFS) are shown in Table 4. We are uncertain why males were more likely to be placed (univariate and multivariate analyses). Sex is not a consistent predictor of discharge destination. ${ }^{16}$ Although younger than females, males had significantly higher rates of falls, urine retention, and BPSD (data not shown). The cumulative effect of these predictors may explain the higher prevalence of nursing home placement among males.

Our study shows that patients who fell had significantly longer lengths of stay. These data support the findings of Vassallo et $\mathrm{al}^{13}$ who found that both single fallers and recurrent fallers in a geriatric nonacute hospital had longer LOS.

Our study has several limitations. First, the population of older patients is larger and more diverse than our single hospital sample and hence our results cannot be extrapolated to all older inpatients. Second, the study population was somewhat unusual in that $52.4 \%$ were born in countries where English is not the predominant language spoken. South-Western Sydney has been settled by successive migrant populations over several decades, most of whom came from non-English-speaking countries. Several such populations have well-established enclaves, some of which offer culturally appropriate services. Third, our findings cannot be generalized beyond older people. Fourth, we treated the CSHA-CFS, an ordinal scale measure, as an interval scale measure and quantitative values should be interpreted cautiously. Fifth, although we found a strong association between falls and nursing home placement, we cannot automatically assume that association equates to causality. However, consistent findings from older nonacute hospital studies ${ }^{12,13}$ and studies conducted in the community ${ }^{18,19,23,24}$ increase the likelihood of a causal relationship between falls and placement. Sixth, while we adjusted for many variables, additional unmeasured factors may have confounded the relationship between falls and nursing home placement. Finally, our selection of predictor variables in the regression models may seem subjective. With the exception of well-established and consistent risk factors in the literature, selection of other predictors for consideration in regression models is always somewhat subjective, depending in part on the population studied and the characteristics of the dataset. While the literature should be used as a guide, inconsistencies in the literature are common. ${ }^{16}$

Despite these limitations, our study has several strengths. Although falls were retrospectively retrieved from an institutional incident reporting database, all other data were ascertained prospectively. Our sample was large, and we studied consecutive patients admitted to our geriatric medicine service during 3 sequential years. Consecutive sampling is the best of all nonprobability samples and, in this broad category, it is most representative of the underlying study base. We adjusted for several potential explanatory variables and included a measure of frailty (CSHA-CFS). Because the CSHA-CFS mixes items such as comorbidity, cognitive impairment, and disability, it appears particularly suited to services encompassing multidisciplinary care. ${ }^{15}$ Due to the nature of its items, the CSHA-CFS may capture some unmeasured variables that may have impacted on function and falls.

Given the financial costs and the predominantly negative experiences associated with nursing home care in Australia and other countries (eg, England), ${ }^{7-9}$ the identification of potentially preventable or remediable risk factors for placement should be a high priority. ${ }^{18}$ The strategies most commonly seen in successful hospital-based fall-prevention trials include detection and prevention of delirium, medication review, patient and staff education, footwear advice, regular toileting schedules, and postfall review. ${ }^{2}$ Most of these are feasible and could readily be incorporated into the care of older people hospitalized with acute illness. Our data show that BPSD, deconditioning, higher levels of frailty, and urine retention are associated with both falls in the hospital and subsequent nursing home placement. Preventing falls among patients with these potentially remediable conditions may reduce the risk of nursing home placement. Strategies targeting these conditions are worthy of further study.

\section{Conclusion}

This study of 2,945 consecutive patients discharged from an acute geriatric medicine service found that falls in the hospital were significantly associated with new placement in a nursing home, irrespective of the absence or presence of injury. A substantial number of falls in the hospital can be prevented. ${ }^{5,6}$ Nursing home care is expensive and, for the most, undesirable. These issues highlight the relevance of this study.

\section{Acknowledgments}

We acknowledge the following geriatricians for their contributions to data collection and data entry: Benyamen Benyamen, Leemin Chan, David Conforti, Rinaldo Gonzales, Angela Khoo, Florence Loh, Colin MacArthur, Milan Simic, and Nalini Thayaparan. 


\section{Disclosure}

The authors report no conflicts of interest in this work.

\section{References}

1. Wright E. 2008-based national population projections for the United Kingdom and constituent countries. Popul Trends. 2010;(139):91-114.

2. Oliver D, Healey F, Haines TP. Preventing falls and fall-related injuries in hospitals. Clin Geriatr Med. 2010;26(4):645-692.

3. Koh SL, Hafizah N, Lee JY, Loo YL, Muthu R. Impact of a fall prevention programme in acute hospital settings in Singapore. Singapore Med $J$. 2009;50(4):425-432.

4. Schwendimann R, Milisen K, Buhler H, De Geest S. Fall prevention in a Swiss acute care hospital setting: reducing multiple falls. $J$ Gerontol Nurs. 2006;32(3):13-22.

5. Hempel S, Newberry S, Wang Z, et al. Hospital fall prevention: a systematic review of implementation, components, adherence, and effectiveness. J Am Geriatr Soc. 2013;61(4):483-494.

6. Miake-Lye IM, Hempel S, Ganz DA, Shekelle PG. Inpatient fall prevention programs as a patient safety strategy: a systematic review. Ann Intern Med. 2013;158(5 Pt 2):390-396.

7. Duffin C. CQC criticises lack of privacy and dignity in England's care homes. Nurs Stand. 2013;27(29):10.

8. Bradshaw SA, Playford ED, Riazi A. Living well in care homes: a systematic review of qualitative studies. Age Ageing. 2012;41(4):429-440.

9. Slama CA, Bergman-Evans B. A troubling triangle. An exploration of loneliness, helplessness, and boredom of residents of a veterans home J Psychosoc Nurs Ment Health Serv. 2000;38(12):36-43.

10. Murray GR, Cameron ID, Cumming RG. The consequences of falls in acute and subacute hospitals in Australia that cause proximal femoral fractures. J Am Geriatr Soc. 2007;55(4):577-582.

11. Foss NB, Palm H, Kehlet H. In-hospital hip fractures: prevalence, risk factors and outcome. Age Ageing. 2005;34(6):642-645.

12. Aditya BS, Sharma JC, Allen SC, Vassallo M. Predictors of a nursing home placement from a non-acute geriatric hospital. Clin Rehabil. 2003; 17(1):108-113.
13. Vassallo M, Sharma JC, Allen SC. Characteristics of single fallers and recurrent fallers among hospital in-patients. Gerontology. 2002; 48(3):147-150.

14. Wieland D, Rubenstein LZ. What do we know about patient targeting in geriatric evaluation and management (GEM) programs? Aging Clin Exp Res. 1996;8(5):297-310.

15. Rockwood K, Song X, MacKnight C, et al. A global clinical measure of fitness and frailty in elderly people. CMAJ. 2005;173(5):489-495.

16. Campbell SE, Seymour DG, Primrose WR. A systematic literature review of factors affecting outcome in older medical patients admitted to hospital. Age Ageing. 2004;33(2):110-115.

17. Inouye SK, Rushing JT, Foreman MD, Palmer RM, Pompei P. Does delirium contribute to poor hospital outcomes? A three-site epidemiologic study. J Gen Intern Med. 1998;13(4):234-242.

18. Tinetti ME, Williams CS. Falls, injuries due to falls, and the risk of admission to a nursing home. $N$ Engl J Med. 1997;337(18):1279-1284.

19. Dunn JE, Furner SE, Miles TP. Do falls predict institutionalization in older persons? An analysis of data from the Longitudinal Study of Aging. J Aging Health. 1993;5(2):194-207.

20. Basic D, Khoo A. Admission variables predicting short lengths of stay of acutely unwell older patients: relevance to emergency and medical short-stay units. Aust Health Rev. 2009;33(3):502-512.

21. Juan A, Salazar A, Alvarez A, Perez JR, Garcia L, Corbella X. Effectiveness and safety of an emergency department short-stay unit as an alternative to standard inpatient hospitalisation. Emerg Med J. 2006; 23(11):833-837.

22. Daly S, Campbell DA, Cameron PA. Short-stay units and observation medicine: a systematic review. Med J Aust. 2003;178(11):559-563.

23. Kiel DP, O'Sullivan P, Teno JM, Mor V. Health care utilization and functional status in the aged following a fall. Med Care. 1991; 29(3):221-228.

24. Wolinsky FD, Johnson RJ, Fitzgerald JF. Falling, health status, and the use of health services by older adults. A prospective study. Med Care. 1992;30(7):587-597.
Clinical Interventions in Aging

\section{Publish your work in this journal}

Clinical Interventions in Aging is an international, peer-reviewed journal focusing on evidence-based reports on the value or lack thereof of treatments intended to prevent or delay the onset of maladaptive correlates of aging in human beings. This journal is indexed on PubMed Central, MedLine,

\section{Dovepress}

CAS, Scopus and the Elsevier Bibliographic databases. The manuscript management system is completely online and includes a very quick and fair peer-review system, which is all easy to use. Visit http://www.dovepress. com/testimonials.php to read real quotes from published authors. 